\title{
LA EDICIÓN DE REVISTAS CIENTÍFICAS EN ESPAÑA: UNA APROXIMACIÓN DESCRIPTIVA
}

\author{
Melba G. Claudio-González* \\ Facultad de Biblioteconomía y Documentación. Universidad de Barcelona. \\ Montserrat Martín-Baranera ${ }^{* *}$ \\ Facultad de Medicina. Universidad Autónoma de Barcelona. \\ Anna Villarroya Planas ${ }^{* * *}$ \\ Facultad de Biblioteconomía y Documentación. Universidad de Barcelona.
}

\begin{abstract}
Resumen: El objetivo principal de este artículo es describir el perfil de las revistas científicas españolas. Los resultados que se presentan proceden de una encuesta a 1.280 revistas científicas españolas incluidas en la base de datos Dulcinea (edición 2012), obteniendo una tasa de respuesta del 43,8\% (n=561). Como objetivos específicos, el estudio se planteó caracterizar las revistas científicas y analizar las peculiaridades de las mismas en torno a una serie de parámetros que conforman las dimensiones económico-financiera, estratégica y operativa de las revistas en el periodo observado. El análisis ha permitido observar cómo el modelo de financiación de las revistas científicas españolas se sostiene principalmente de las ayudas institucionales, cómo la mayoría de las revistas carece de una estructura organizativa estable y depende, en gran medida, del trabajo voluntario y cómo el debate sobre las limitaciones y dificultades que afectan al desarrollo del acceso abierto sigue vigente.

Palabras clave: revistas científicas; España; financiación; acceso abierto.
\end{abstract}

Title: THE SPANISH SCIENTIFIC JOURNALS: A DESCRIPTIVE APPROACH.

Abstract: The main objective of this article is to present a broad description of Spanish scientific journals. The results were obtained from a survey distributed to 1,280 editors of Spanish scientific journals included in Dulcinea database (2012 edition). The response rate was 43,8\%. As specific objectives, the study aimed to characterize scientific journals and to analyze the particularities of a set of parameters that make up the economic, strategic and operational dimensions of the journals in the period studied. The results show that the funding model of the Spanish scientific journals depends mainly on institutional support, that many journals lack a stable organizational structure and rely heavily on volunteer work, and that the debate on the limitations and difficulties affecting the development of open access remains open.

Keywords: scientific journals; Spain; financing; open access.

\section{INTRODUCCIÓN}

Pensar en la investigación sobre la edición de revistas científicas evoca los principales elementos disruptivos de carácter tecnológico, social, político o económico que han marcado la investigación de finales del siglo XX e inicios del XXI. Entre ellos, la adaptación a los cambios asociados al desarrollo de la filosofía del acceso abierto (Crow y Goldstein, 2003; Chi Chang, 2006; Lamberti y Solaro, 2011; Dai y otros, 2014; Das, 2015; Schimmer y otros, 2015); las discusiones y propuestas en torno a la viabilidad financiera de las revistas (Björk y Hedlund, 2009; Crow, 2009; Houghton y otros, 2009; Morrison, 2013); los debates en torno a los índices de calidad o de impacto (Bordons y otros, 2002; Björk y Solomon, 2012; Riera y Aibar, 2013); la preocupación por la visibilidad y el posicionamiento internacional de las revistas científicas (Buela Casal, 2001; Abadal y Rius Alcaraz, 2008; Aréchaga, 2009; RodríguezYunta, 2015) y, más recientemente, el impacto de los requerimientos de la ley y las normativas universitarias que obligan a poner a disposición pública los resultados de la investigación financiada con fondos públicos y otros desafíos políticos de la ciencia abierta (Friend, 2011; Abadal y otros, 2013; Rodríguez-Yunta y Giménez-Toledo, 2013; Alonso y Echevarría, 2014; García Arístegui y Rendueles, 2014).

Los estudios en este ámbito han pivotado sobre al menos una de dichas cuestiones, ya sea con miras a censar y caracterizar las revistas o a retratar los logros, barreras y fortalezas de la producción científica en un determinado período. Con intereses tan distintos en el centro de las investigaciones, no es de extrañar que las miradas sobre la

\footnotetext{
*melbaclaudio@gmail.com

** montserrat.martin@uab.cat

*** annavillarroya@ub.edu
}

Recibido: 08-08-2016; $2^{\mathrm{a}}$ versión: 07-12-2016; aceptado: 16-01-2017.

CLAUDIO GONZÁLEZ, M.G., MARTÍN BARANERA, M. y VILLARROYA PLANAS, A. La edición de revistas científicas en España: una aproximación descriptiva. Anales de Documentación, 2017, vol. 20, $\mathrm{n}^{\mathrm{o}} 1$. Disponible en: http://dx.doi.org/10.6018/analesdoc.20.1.265771. 
producción científica nacional sean tan diversas como los objetivos que han motivado los intentos de contabilizar y obtener un perfil general de las revistas científicas españolas. No obstante, los esfuerzos empíricos han contribuido a constituir un reflejo de la conformación y consolidación de una disciplina científica en un momento determinado (Abad-García y otros, 2015), han ampliado el conocimiento sobre el impacto y la visibilidad de las revistas científicas españolas (Bordons y Gómez Caridad, 1997; Bordons y otros, 2002; Zamora y otros, 2007; Abadal y Rius Alcaraz, 2008; Osca-Lluch y otros, 2008), así como de los distintos modelos de publicación científica (Melero y Abad-García, 2008; Rodrigues y Abadal, 2014) y han ayudado a desvelar tendencias y debates emergentes sobre las prácticas editoriales de las revistas científicas en España (Urdín Caminos, 2001; Osca-Lluch y otros, 2008; García, 2015).

En este marco, el objetivo principal de este artículo es describir el perfil de las revistas científicas españolas. Como objetivos específicos, se plantean, por un lado, la caracterización de las revistas científicas españolas y, por otro, el análisis de las peculiaridades de las mismas en torno a una serie de parámetros que conforman las dimensiones económico-financiera, estratégica y operativa de las revistas en el periodo observado. En la línea planteada por Villarroya y otros, 2012, estos parámetros permitirían una aproximación a los modelos de negocio de las revistas científicas españolas. Con el fin de dar respuesta a estos objetivos el trabajo se plantea las siguientes preguntas de investigación:

PI1: ¿Cuáles son las principales características de las revistas científicas españolas?

PI2: ¿Existe una estructura de financiación imperante entre las revistas científicas españolas?

PI3: ¿Cuáles son los principales gastos de las revistas científicas españolas y en qué área de la actividad editorial recae el mayor peso de sus costes?

PI4: ¿En qué situación económica se encuentran la mayoría de las revistas científicas españolas?

PI5: ¿Cuál es el tipo de vinculación y la dedicación horaria del personal de las revistas científicas españolas?

PI6: ¿Qué balance hacen las revistas científicas españolas de su experiencia con el acceso abierto?

PI7: ¿Qué factores son los que podrían afectar, de una manera más importante, el funcionamiento de las revistas científicas españolas en un futuro cercano?

\section{METODOLOGÍA}

Los resultados de este trabajo proceden de una encuesta auto administrada vía Limesurvey y distribuida entre 1.280 editores de revistas científicas españolas incluidas en la base de datos Dulcinea, edición 2012 $<$ http://www.accesoabierto.net/dulcinea $>$, que recoge los derechos de copyright y las condiciones de autoarchivo de las revistas españolas (Melero y otros, 2009).

Con la idea de obtener una visión general de la arquitectura del servicio que ofrecen las revistas, el cuestionario incluyó un total de 28 preguntas ordenadas en cinco apartados diferenciados: datos descriptivos de la revista; dimensión económico-financiera; dimensión estratégica; dimensión operativa y perspectivas de futuro. El Apéndice A muestra el detalle de los parámetros específicos que se observaron dentro de cada una de las tres dimensiones del modelo de negocio de las revistas.

El marco temporal del estudio abarcó el ejercicio de la actividad editorial de los tres años concluidos justo antes de la administración de la encuesta (2009-2011).

Una vez consensuada una versión completa del cuestionario, se realizó una prueba piloto para confirmar que los conceptos, indicadores y variables seleccionadas se adecuaban al vocabulario y conocimientos generales de los responsables de las revistas. La prueba piloto constó de tres partes: una primera consulta a expertos, entre los que se incluyó a cinco personas con amplia experiencia en métodos de investigación y diseño de cuestionarios, así como en el ámbito de la edición de revistas científicas. Una segunda que consistió en la administración del cuestionario a una muestra reducida de sujetos cuyo perfil se correspondía con el de la población a observar y una tercera, consistente en la administración del cuestionario en línea a una muestra reducida de sujetos, siguiendo las mismas pautas de administración del cuestionario que posteriormente serían utilizadas. La prueba se realizó con sujetos que respondían al mismo perfil de la población a estudiar y que no habían participado en las fases iniciales de la prueba piloto. El tiempo estimado de respuesta que se obtuvo a partir de dicha prueba fue de 17 minutos.

Finalmente, una vez depurada la base de datos, se envió la invitación a participar en la encuesta aplicando las propuestas del método Dillman (Dillman y otros, 2009). Siguiendo las recomendaciones de dichos autores, se establecieron una serie de acciones de seguimiento de las respuestas al cuestionario que requirió varios contactos para lograr una tasa de respuesta alta. 
La encuesta, que se mantuvo abierta entre los meses de noviembre de 2012 y marzo de 2013, obtuvo una tasa de respuesta del 44\% $(n=561)$. Al cierre de la encuesta, la mediana resultante del tiempo de respuesta se situó en 18 minutos, muy cercana a los 17 minutos estimados en la prueba piloto.

Para el análisis estadístico de los datos se utilizó el programa SPSS-22.0. Las variables categóricas se describieron con frecuencias absolutas y porcentajes y las variables cuantitativas con sus respectivas medias y desviaciones estándar, o bien medianas y valores mínimo y máximo.

En el análisis de variables categóricas se aplicó el test de chi-cuadrado o, como alternativa, el test exacto de Fisher cuando los efectivos esperados en al menos una celda fueron inferiores a cinco. En algunos casos, las variables se expresaron en escala de tipo Likert. En el análisis de variables cuantitativas, se aplicó un análisis de la variancia, o bien el test de Kruskal-Wallis como alternativa no paramétrica.

\section{RESULTADOS}

\subsection{Distribución de las revistas por comunidad autónoma}

Madrid, Cataluña y Andalucía aglutinaron el 58\% de la publicación de revistas científicas en España, mientras que aproximadamente el $27 \%$ de la producción se concentró en la Comunidad Valenciana, Castilla y León, País Vasco, Región de Murcia y Galicia.

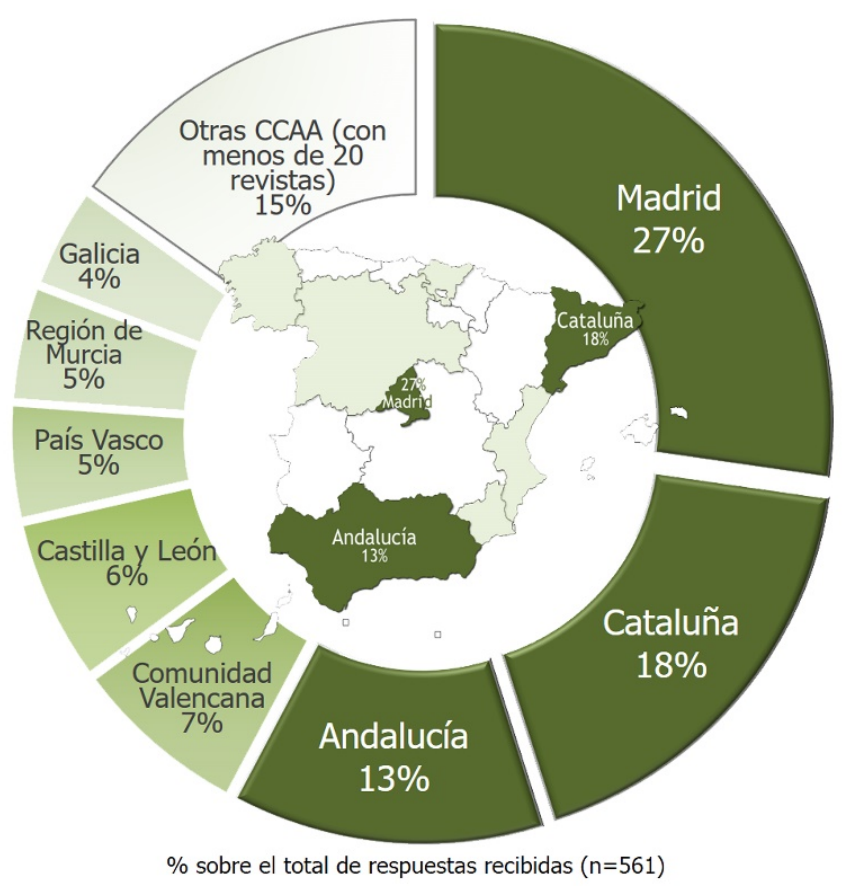

Figura 1. Distribución de las revistas por comunidad autónoma.

\subsection{Distribución de las revistas por tipo de entidad propietaria}

Se observó un predominio de las revistas de titularidad universitaria $(37 \% ; n=207)$, que conjuntamente con las asociaciones y sociedades científicas y los organismos de investigación, agrupan el $80 \%(\mathrm{n}=451)$ del total de las revistas científicas españolas. 


\begin{tabular}{l|r|r}
\hline Tipo de entidad propietaria & $\mathbf{n}$ & $\%$ \\
\hline Editorial Universitaria & 207 & 36,9 \\
\hline Asociación o sociedad profesional & 152 & 27,1 \\
\hline Organismo de investigación público (OPI) & 92 & 16,4 \\
\hline Editorial comercial & 38 & 6,8 \\
\hline Administración pública & 22 & 3,9 \\
\hline Varias & 50 & 8,9 \\
\hline Total & 561 & 100,0 \\
\hline
\end{tabular}

Tabla I. Distribución de las revistas por tipo de entidad propietaria.

\subsection{Distribución de las revistas por rama de conocimiento}

En la distribución de las revistas por rama de conocimiento predominaron las de Artes y humanidades y Ciencias sociales y jurídicas, que aglutinan el $68 \%(\mathrm{n}=380)$ del total de las revistas.

\begin{tabular}{|c|c|c|}
\hline Rama de conocimiento & $\mathrm{n}^{0}$ & \% válido \\
\hline Artes y humanidades & 191 & 34,0 \\
\hline Ciencias sociales y jurídicas & 189 & 33,7 \\
\hline Ciencias de la salud & 75 & 13,4 \\
\hline Ciencias & 57 & 10,2 \\
\hline Multidisciplinar & 28 & 5,0 \\
\hline Ingeniería y arquitectura & 21 & 3,7 \\
\hline Total & 561 & 100,0 \\
\hline \multicolumn{3}{|c|}{$\begin{array}{l}\text { " La referencia utilizada para delimitar las ramas de conocimiento ha sido la la } \\
\text { clasificación oficial de la Enseñanza Universitaria en España (Real Decreto } \\
1618 / 2011, \text { de } 14 \text { de noviembre, sobre reconocimiento de estudios en el } \\
\text { ámbito de la Educación Superior). }\end{array}$} \\
\hline
\end{tabular}

Tabla II. Distribución de las revistas por rama de conocimiento.*

\subsection{Formato de la revista}

Casi la mitad de las revistas se publicaron tanto en formato digital como impreso (47\%; n=266). El 33\% ( $\mathrm{n}=184)$ se publicó únicamente en formato digital y el $20 \%(n=111)$, en formato impreso.

\subsection{Idioma}

En la distribución por idioma, el mayor grupo de revistas publicó en castellano o en inglés en proporciones variables $(42 \% ; n=233)$. Un 17\% $(n=93)$ publicó sólo en castellano, un $5 \%(n=29)$ sólo en inglés y un 3\% publicó la revista íntegramente en ambos idiomas (castellano e inglés). Las revistas restantes se dividen entre un $16 \%$ ( $\mathrm{n}=90)$ que informó publicar en cuatro idiomas o más en proporciones variables y un $18 \%(n=99)$ que informó que combinaba el castellano con las lenguas cooficiales u otros idiomas.

Por otra parte, el idioma se asoció de forma estadísticamente significativa con la rama de conocimiento $(\mathrm{p}<0,0005)$. La Figura 2 muestra un porcentaje mayor de revistas en inglés entre las correspondientes a las áreas de Ciencias, tecnología, ingeniería y matemáticas $(16,7 \% ; n=13)$, siendo también en estas áreas donde predominan las revistas que combinan artículos en inglés o castellano en proporciones variables $(55,1 \% ; n=43)$. En las ramas de Ciencias Sociales y Humanidades se observa un predominio de las revistas que publican en castellano o inglés (41,1\%; $\mathrm{n}=156)$, así como de las de carácter multilingüe $(38,9 \% ; n=148)$. 


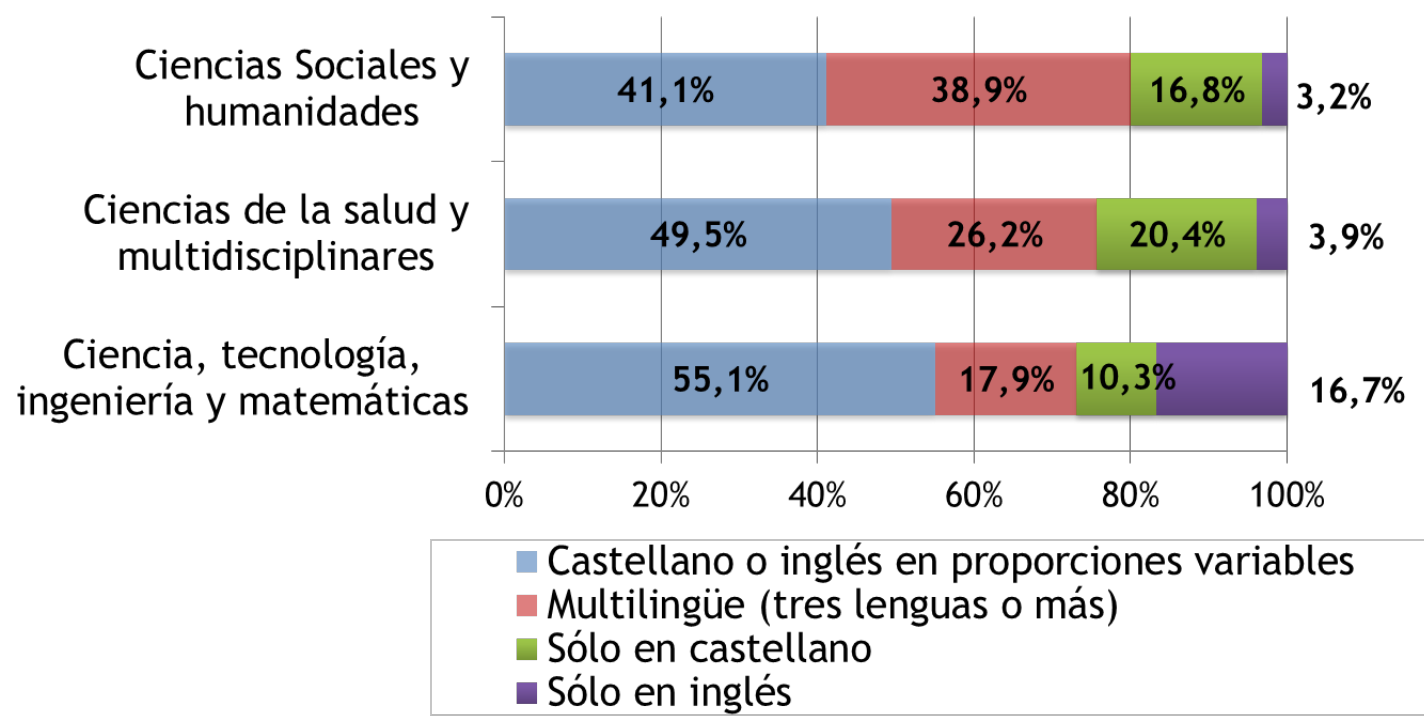

Nota: Con el objetivo de aplicar un test estadístico para explorar relaciones entre el idioma y el área de conocimiento de las revistas, se reagruparon las áreas de conocimiento originales y el idioma de las revistas.

Figura 2. Distribución de idiomas por rama de conocimiento.

\subsection{Estructura de la financiación de las revistas científicas españolas}

Los ingresos monetarios más frecuentes entre las revistas fueron los procedentes de la Administración Pública u otras entidades que apoyan la producción científica. Así, un 70\% (n=391) de los encuestados informó percibir alguno de los tipos de ingresos incluido dentro de dicha categoría general, mientras que la mitad informó percibir algún tipo de ingreso proveniente de transacciones directas tales como suscripciones individuales o suscripciones institucionales (véase Figura 3).

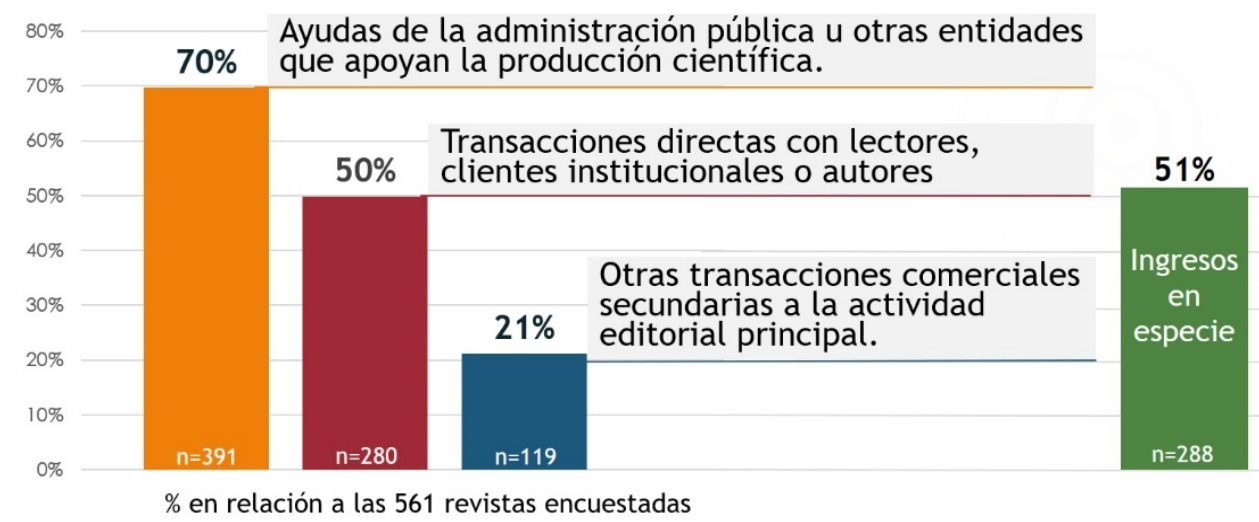

Figura 3. Porcentaje de revistas con ingresos de cada categoría general.

Con una tasa de respuesta del $92 \%(n=518)$, los resultados detallados que se presentan a continuación muestran los porcentajes en relación a las 518 revistas que respondieron a todas las preguntas relacionadas con las fuentes de ingresos.

Aproximadamente la mitad de las revistas que declararon percibir ingresos de ayudas institucionales recibió ayudas directas de la entidad propietaria $(48 \% \mathrm{n}=246)$ y un $32 \%(\mathrm{n}=167)$ obtuvo subvenciones de la Administración Pública. Sólo el 19\% $(\mathrm{n}=99)$ informó percibir ingresos vía patrocinios privados y apenas el 6\% $(\mathrm{n}=30)$ recibió donativos.

Más del $47 \%(\mathrm{n}=244)$ de las revistas informaron percibir ingresos a través de suscripciones individuales y poco más del $40 \%(\mathrm{n}=211)$, vía suscripciones institucionales. Solo el $6 \%(\mathrm{n}=33)$ de las revistas percibió ingresos por pagos por uso y un $3 \%$ cobró cuotas a los autores por publicar (APC). 
Dentro de las transacciones secundarias a la actividad principal destacan, con un peso del 13\% ( $\mathrm{n}=67)$, los ingresos por publicidad percibidos mayoritariamente por asociaciones o sociedades profesionales. Un 10\% (54) de las revistas percibió ingresos en concepto de coorganización de conferencias o congresos y solo un $2 \%$ ( $\mathrm{n}=12)$ recibió ingresos procedentes de consultorías y servicios a terceros, siendo las revistas universitarias las principales destinatarias de este tipo de ingresos.

Más de la mitad de las revistas $(56 \% \mathrm{n}=288)$ informó recibir algún tipo de ingreso en especie, ya fuera en forma de equipos informáticos, espacios o apoyo administrativo. El porcentaje fluctuó entre un $36 \%$ de revistas que recibieron apoyo administrativo y un $45 \%$ que recibió ayudas en forma de equipos informáticos, material fungible, etc., o cesión de espacios o mobiliario.

Al explorar en qué tipo de ingresos monetarios recaía el mayor peso del soporte financiero de las revistas, la tasa de respuesta se situó en el 86\% $(\mathrm{n}=482)$. Cada una de esas 482 revistas indicó cómo se distribuía el 100\% de sus ingresos, adjudicando el porcentaje correspondiente a las distintas fuentes de ingresos (véase Tabla III).

\begin{tabular}{l|l|l|l|l|l|l|l|l|l|c}
\hline $\begin{array}{l}\text { Fuente de ingreso } \\
\text { (Fdl) 2009-2011 }\end{array}$ & Media & $\begin{array}{c}\text { Desviación } \\
\text { estándar }\end{array}$ & $\begin{array}{c}\text { Percentil } \\
\mathbf{2 5}\end{array}$ & Mediana & $\begin{array}{c}\text { Percentil } \\
75\end{array}$ & Mínimo & Máximo & Moda & $\mathrm{n}$ & $\begin{array}{c}\% \\
\text { Total }\end{array}$ \\
\hline $\begin{array}{l}\text { Entidades apoyo } \\
\text { producción } \\
\text { cientifica }\end{array}$ & 73,36 & 31,68 & 50,0 & 90,0 & 100,0 & 5 & 100 & 100 & 255 & 45,5 \\
\hline $\begin{array}{l}\text { Suscriptores } \\
\text { institucionales }\end{array}$ & 36,19 & 33,36 & 10,0 & 20,0 & 60,0 & 1 & 100 & 10 & 205 & 36,5 \\
\hline $\begin{array}{l}\text { Ingresos } \\
\text { monetarios } \\
\text { provenientes de } \\
\text { los Lectores }\end{array}$ & 36,50 & 32,81 & 10,0 & 25,0 & 67,5 & 1 & 100 & 10 & 164 & 29,2 \\
\hline $\begin{array}{l}\text { Otras } \\
\text { transacciones } \\
\text { comerciales } \\
\text { secundarias a la } \\
\text { actividad principal }\end{array}$ & 39,24 & 34,78 & 10,0 & 30,0 & 70,0 & 1 & 100 & 5 & 50 & 8,9 \\
$\begin{array}{l}\text { Ingresos } \\
\text { monetarios } \\
\text { provenientes de } \\
\text { los Autores }\end{array}$ & 27,71 & 22,40 & 10,0 & 20,0 & 40,0 & 10 & 90 & 10 & 14 & 2,5 \\
\hline
\end{tabular}

Tabla III. Porcentaje del total de ingresos que representa cada una de las principales fuentes de ingresos.

Los resultados muestran un predominio de los ingresos procedentes de las ayudas institucionales (ya sea de la Administración Pública u otras entidades de apoyo a la producción científica) con una mediana del 90\%. La mediana de los otros tres tipos de ingresos fluctuó entre un 20\% (en el caso de las suscripciones institucionales e ingresos provenientes de autores) y un $30 \%$ (en el caso de transacciones comerciales secundarias a la actividad principal).

Cabe destacar también que para el $47 \%$ de las revistas que recibieron ayudas institucionales, éstas representaron más del $90 \%$ del total de sus ingresos.

Con el objetivo de ver la existencia o no de diferencias en la distribución de la financiación de las revistas según la rama de conocimiento se llevó a cabo un análisis exploratorio que puso de relieve cómo entre el 70 y el $79 \%$ de las revistas recibía ayudas institucionales, independientemente de la rama de conocimiento. En cualquiera de las ramas, las transacciones directas resultaron ser la segunda fuente de ingresos más frecuente en las tres categorías consideradas, oscilando entre un 52\% en el área de Ciencias Sociales y Humanidades y un 64\% en el área de Ciencia, Tecnología, Ingeniería y Matemáticas. Únicamente en el caso de los ingresos monetarios procedentes de otras transacciones comerciales secundarias a la actividad principal se observaron diferencias significativas por áreas de conocimiento. Concretamente, fue en las revistas de Ciencias de la Salud y multidisciplinares donde se observó una mayor frecuencia (45\%) de ingresos generados por dicho concepto (véase Figura 4). 


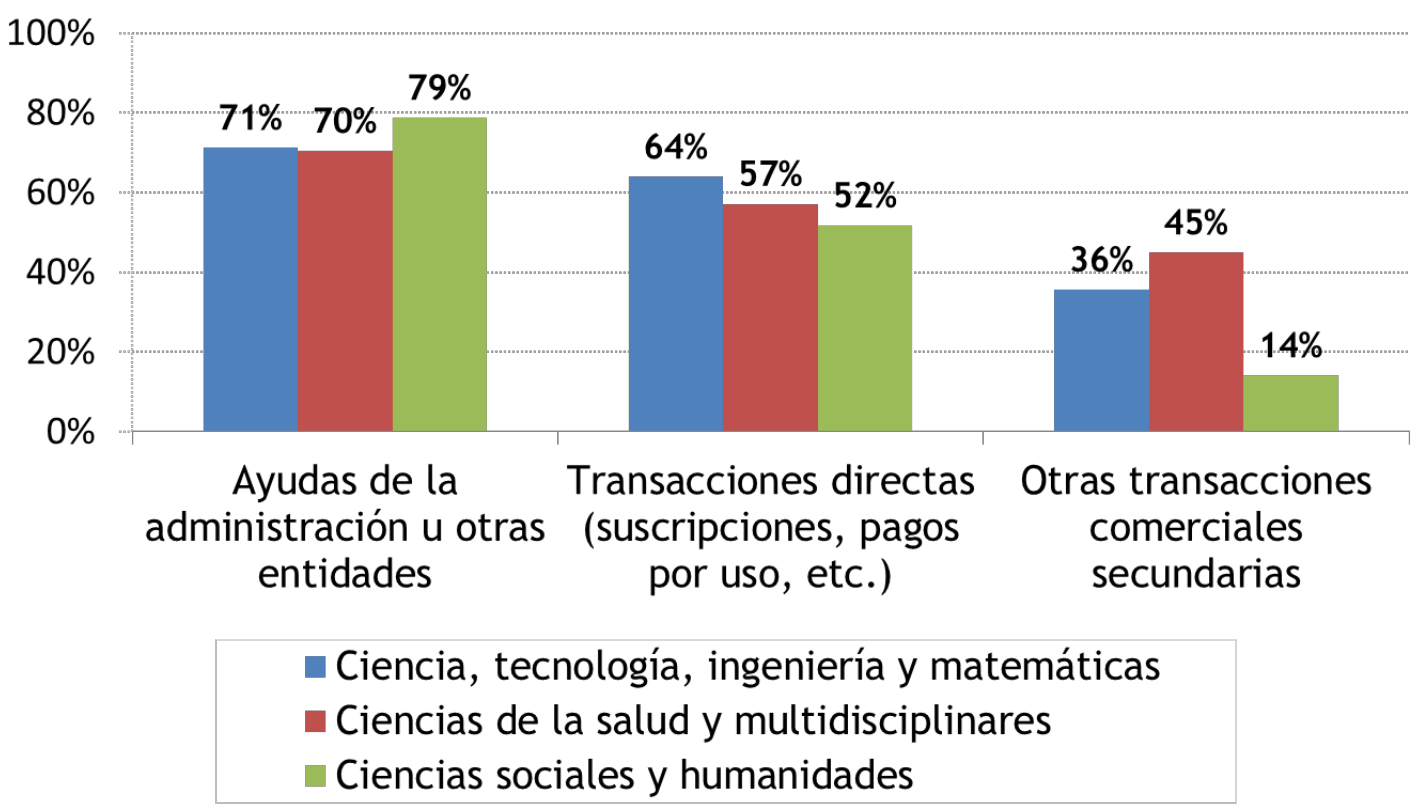

Nota: Con el objetivo de aplicar un test estadístico para explorar relaciones entre la distribución de la financiación de las revistas y el área de conocimiento, se ha llevado a cabo una reagrupación de las áreas de conocimiento originales.

Figura 4. Porcentaje de revistas con ingresos de cada categoría general según rama de conocimiento.

Por último, con el objetivo de ver si existían diferencias entre la estructura de la financiación de las revistas y su inclusión en bases de datos internacionales (Web of Science y Scopus) se llevó a cabo un análisis exploratorio para un conjunto de revistas en el que se observó que la estructura de financiación de las mismas no difería significativamente en función de su circulación o no en bases de datos internacionales, con la única excepción de los ingresos monetarios generados de suscripciones, uso o cuotas, que estaban presentes en el $68 \%$ de las incluidas en dichas bases frente a un $54 \%$ de las no incluidas $(\mathrm{p}=0,031)$.

\subsection{Distribución de los principales costes}

Atendiendo a la distribución más frecuente del total de los costes entre las cuatro áreas generales de la actividad editorial, los resultados mostraron un mayor peso de los costes relativos a las actividades de producción, con una mediana del $80 \%$. Este resultado se situó muy por encima de los costes relacionados con las otras tres áreas: creación de contenidos (mediana=25\%), gestión editorial (mediana=15\%) y distribución (mediana=13\%).

\begin{tabular}{|c|c|c|c|c|c|c|c|c|c|}
\hline Área de actividad editorial & Media & $\begin{array}{l}\text { Desviación } \\
\text { estándar }\end{array}$ & \begin{tabular}{|c|} 
Percentil \\
25 \\
\end{tabular} & Mediana & $\begin{array}{c}\text { Percentil } \\
75\end{array}$ & Minimo & Máximo & $\mathrm{n}$ & $\begin{array}{c}\% \\
\text { Total }\end{array}$ \\
\hline $\begin{array}{l}\text { Producción: costes asociados a la } \\
\text { maquetación (digital o papel), } \\
\text { impresión, encuadernación, etc. }\end{array}$ & 71,45 & 25,26 & 50,00 & 80,00 & 95,00 & 5 & 100 & 424 & 75,6 \\
\hline $\begin{array}{l}\text { Creación de contenidos: costes } \\
\text { asociados a la preparación del } \\
\text { contenido editorial de la } \\
\text { publicación. }\end{array}$ & 30,55 & 23,88 & 10,00 & 25,00 & 40,00 & 1 & 100 & 183 & 32,6 \\
\hline $\begin{array}{l}\text { Gestión editorial: costes de } \\
\text { comercialización, ventas de } \\
\text { publicidad, finanzas y } \\
\text { administración, incluidos los gastos } \\
\text { de gestión y de las oficinas que } \\
\text { llevan a cabo dichas actividades. }\end{array}$ & 22,71 & 21,25 & 10,00 & 15,00 & 30,00 & 1 & 100 & 204 & 36,4 \\
\hline $\begin{array}{l}\text { Distribución: Costes de } \\
\text { distribución, incluyendo todos los } \\
\text { costes de mantenimiento de } \\
\text { archivos de abonados y el servicio } \\
\text { al cliente. }\end{array}$ & 18,95 & 17,46 & 10,00 & 13,00 & 20,00 & 3 & 100 & 247 & 44,0 \\
\hline
\end{tabular}

Tabla IV. Porcentaje del total de costes que representan las partidas asociadas a cada una de las áreas de la actividad editorial. 


\subsection{Distribución de los principales gastos}

El análisis de los gastos se realizó a partir del desglose de los mismos entre bienes corrientes y suministros (materias primas y otros aprovisionamientos), bienes de capital fijo (reposición de equipamientos, infraestructuras, etc.), personal y pagos a revisores. Otros gastos externos se incluyeron en una categoría que recogía cualquier otro servicio contratado externamente distinto de los pagos a revisores.

Los resultados muestran cómo la partida de servicios externos, además de haber sido la más frecuente (un $42 \%$ de las revistas informaron tener gastos por este concepto), es la que tiene un mayor peso en el total de gastos de las revistas científicas españolas con una mediana del $80 \%$. Le siguieron los gastos de personal (con una mediana del $50 \%$ ) y los gastos en bienes corrientes (mediana $=40 \%$ ). Los gastos con menor peso correspondieron a las partidas de bienes de capital fijo (informados por el $18 \%$ de los encuestados, con una mediana de un $25 \%$ ) y a los pagos a revisores (informados por solo el $6 \%$ de los encuestados, con una mediana de un $20 \%$ ).

\begin{tabular}{l|c|r|r|r|r|r|r|r|c}
\hline $\begin{array}{l}\text { Conceptos } \\
\text { de gastos }\end{array}$ & Media & $\begin{array}{c}\text { Desviación } \\
\text { estándar }\end{array}$ & $\begin{array}{c}\text { Percentil } \\
\mathbf{2 5}\end{array}$ & Mediana & $\begin{array}{c}\text { Percentil } \\
75\end{array}$ & Mínimo & Máximo & $\mathrm{n}$ & $\begin{array}{c}\% \\
\text { Total }\end{array}$ \\
\hline $\begin{array}{l}\text { Gastos en } \\
\text { servicios } \\
\text { externos }\end{array}$ & 66,53 & 34,49 & 30,00 & 80,00 & 100,00 & 5 & 100 & 235 & 41,9 \\
\hline Personal & 52,27 & 33,23 & 20,00 & 50,00 & 85,00 & 1 & 100 & 164 & 29,2 \\
\hline $\begin{array}{l}\text { Gastos en } \\
\text { bienes } \\
\text { corrientes }\end{array}$ & 50,31 & 38,14 & 10,00 & 40,00 & 100,00 & 1 & 100 & 186 & 33,2 \\
\hline $\begin{array}{l}\text { Pagos a } \\
\text { revisores } \\
\text { externos }\end{array}$ & 20,82 & 22,43 & 10,00 & 10,00 & 20,00 & 5 & 100 & 34 & 6,1 \\
\hline $\begin{array}{l}\text { Gastos en } \\
\text { bienes de } \\
\text { capital fijo }\end{array}$ & 22,60 & 25,15 & 10,00 & 10,00 & 25,00 & 1 & 100 & 103 & 18,4 \\
\hline
\end{tabular}

Tabla V. Porcentaje del total de gastos que representan las partidas asociadas a cada concepto general.

\subsection{Situación económica de las revistas científicas españolas}

La tasa de respuesta sobre el resultado económico de la revista al cierre de los últimos tres años naturales previos a la encuesta se situó en torno al 57\%. Para cada uno de los tres años, cerca del $62 \%$ declaró haber obtenido un resultado de equilibrio financiero y un 33\% informó haber finalizado con pérdidas. Por último, un 6\% informó ganancias durante los años 2009 y 2010, y un 4\% en el 2011.

\begin{tabular}{|c|c|c|c|c|}
\hline Año & Pérdida & Equilibrio & Beneficios & Total \\
\hline $2009 \quad n$ & 102 & 192 & 18 & 312 \\
\hline$\%$ sobre $n=312$ & 32,7 & 61,5 & 5,8 & $100 \%$ \\
\hline 2010 & 104 & 197 & 18 & 319 \\
\hline$\%$ sobre $n=319$ & 32,6 & 61,8 & 5,6 & $100 \%$ \\
\hline 2011 & 106 & 203 & 13 & 322 \\
\hline$\%$ sobre $n=322$ & 32,9 & 63,0 & 4,0 & $100 \%$ \\
\hline
\end{tabular}

Tabla VI. Resultado económico de los últimos tres años.

También se exploró la evolución de los resultados económicos. Para ello, se seleccionaron sólo las revistas que respondieron la pregunta los tres años $(55 \%$; $=307)$. Así, el 7\% de las 307 revistas informó resultados económicos variables mientras que más del $90 \%$ de éstas cerró los tres años con un mismo resultado económico. El equilibrio financiero es el resultado más frecuente $(59 \% ; n=180)$, seguido de un $31 \%(n=94)$ de revistas con pérdidas los tres años. Solo un $4 \%$ informó haber cerrado los tres años con beneficios. 
Se observó una relación positiva entre los ingresos percibidos en concepto de suscripciones institucionales y el resultado económico obtenido por las revistas entre 2009 y 2011. La Tabla VII muestra que el 75\% de las revistas que contaban con ingresos por suscripciones institucionales obtuvieron un resultado económico de equilibrio financiero o beneficio.

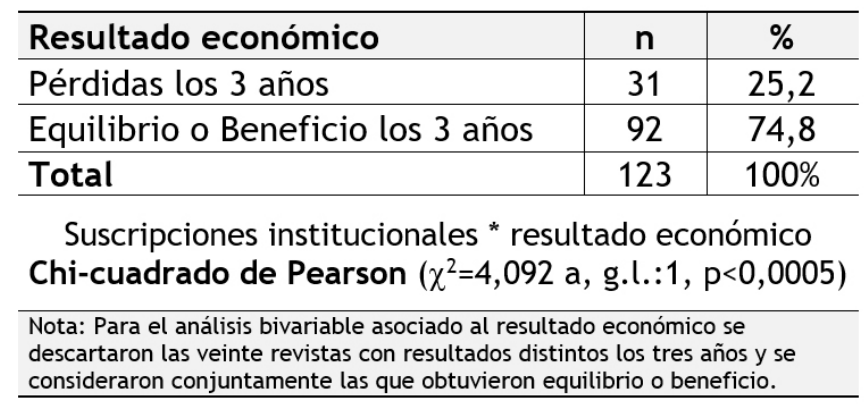

Tabla VII. Relación entre el resultado económico agregado y el tipo de ingresos monetarios generados de suscripciones institucionales.

Finalmente, se comparó el perfil general de las revistas que contestaron y las que no contestaron a las preguntas de resultados económicos. No se encontraron diferencias significativas entre unas y otras en lo que respecta a aspectos tales como la rama de conocimiento o la existencia o no de personal propio. Sin embargo, sí se observaron diferencias estadísticamente significativas entre las revistas que percibieron ingresos monetarios procedentes de transacciones directas (tales como suscripciones individuales o institucionales) y de otras transacciones comerciales. Ambos tipos de ingresos resultaron ser más frecuentes entre las revistas que contestaron que entre las que no contestaron a las preguntas de resultado económico $(\mathrm{p}<0,0005$ y $\mathrm{p}=0,001$ respectivamente). Asimismo, también se observaron diferencias según el tipo de editorial $(\mathrm{p}=0,008)$, especialmente en el caso de las asociaciones o sociedades profesionales, en las que un $67,8 \%$ contestaron a las preguntas.

\subsection{Estructura organizativa}

El análisis reveló un predominio del personal voluntario en las revistas científicas españolas, hallándose presente en más del $70 \%$ de las mismas y siendo el personal con mayor dedicación horaria con una mediana de la Equivalencia a Jornada Completa (EJC) $=1,25$ (véase Tabla VIII).

\begin{tabular}{r|r|r|r|r}
\hline Tipo de personal: & voluntario & propio & externo & becario \\
\hline $\mathrm{n}=$ & 395 & 220 & 143 & 94 \\
\hline $\begin{array}{r}\text { \% sobre el total de respuestas } \\
(\mathrm{n}=511)\end{array}$ & $77,3 \%$ & $43,1 \%$ & $28,0 \%$ & $18,4 \%$ \\
\hline Mediana $=$ & $\mathbf{1 , 2 5}$ & $\mathbf{0 , 7 5}$ & $\mathbf{0 , 5 0}$ & $\mathbf{0 , 5 0}$ \\
\hline Mínimo $=$ & 0,25 & 0,25 & 0,25 & 0,25 \\
\hline Máximo $=$ & 15,00 & 7,5 & 9,25 & 8,75 \\
\hline
\end{tabular}

Tabla VIII. Descriptivos estadísticos de las equivalencias a jornada completa (EJC) de la dedicación de los recursos humanos según el tipo de vinculación.

También se observó una relación estadísticamente significativa entre el tipo de entidad propietaria y las EJC del personal propio y del personal voluntario. Así, la Administración Pública fue el tipo de entidad con mayor dedicación horaria del personal propio, con una mediana de la EJC próxima a una jornada completa. Las asociaciones y sociedades profesionales, junto a las editoriales comerciales, mostraron la mayor dedicación horaria del personal voluntario, también con una mediana de la $\mathrm{EJC}=1,00$.

\subsection{Postura ante el acceso abierto}

El 59\% $(n=333)$ de los encuestados declaró tener experiencia con el acceso abierto y el 13\% $(n=71)$, estar inmerso en el proceso de conversión. El $80 \%$ de las revistas con experiencia con el acceso abierto la valoró en términos positivos o muy positivos. Entre las posibles dificultades para adoptar el acceso abierto, un 45\% identificó las limitaciones financieras, un $31 \%$, las limitaciones en la estructura organizativa y solo un $1 \%$ manifestó no tener ninguna dificultad. Entre los aspectos positivos del acceso abierto, los encuestados identificaron mayoritariamente la visibilidad, el favorecer el acceso a la ciencia, así como el aumento de lectores, visitas y descargas. Entre los 
argumentos desfavorables a la experiencia, destacaron su escasa viabilidad, incidiendo en que la financiación de las revistas de acceso abierto es débil.

\subsection{Oportunidades y amenazas para la edición de revistas científicas en España}

Entre los factores identificados como oportunidades por la mayoría de encuestados destacan las tecnologías de la información y la comunicación, reconocidas como una Gran oportunidad por el 76\% (n=374) de quienes valoraron dicha opción; y las modificaciones en las normativas relacionadas con el acceso abierto, valorada como Gran oportunidad por el $47 \%(\mathrm{n}=206)$ de quienes valoraron dicha opción. Del lado de los factores identificados como amenazas, solo destacó el impacto de las multinacionales en el sector, valorado por un 64\% ( $\mathrm{n}=357)$ de los encuestados. Entre éstos, más de la mitad (54\%) las consideró como una Gran amenaza o una Amenaza.

En cuanto a las principales ventajas competitivas que podrían ayudar a las revistas a posicionarse mejor en el mercado editorial o a contribuir a la sostenibilidad de la actividad editorial en general, gran parte de los encuestados coincidieron en destacar las Habilidades y competencias del equipo directivo (67\%), seguidas del Prestigio del comité editorial (63\%) y del Factor de impacto de la revista (62\%).

\section{DISCUSIÓN Y CONCLUSIONES}

A partir de un análisis descriptivo por encuesta dirigido a los responsables de las revistas científicas españolas incluidas en la base de datos Dulcinea se ha conseguido tener una caracterización básica del perfil de las mismas, así como información, hasta ahora inédita, sobre diversas dimensiones del proceso de gestión editorial de las mismas.

En cuanto al perfil de las revistas científicas españolas, se ha visto cómo Madrid, Cataluña y Andalucía siguen siendo las regiones que editan la mayoría de las revistas científicas españolas, coincidiendo con investigaciones previas (Bordons y Gómez Caridad, 1997; Urdín Caminos, 2001; Bordons y otros, 2002; Zamora y otros, 2007; OscaLluch y otros, 2008).

En cuanto a la titularidad, el análisis ha puesto de relieve un predominio de revistas universitarias y, en menor medida, de revistas de asociaciones y sociedades profesionales. Dicho resultado coincide con los estudios referenciados con anterioridad, en los cuales aparecen las universidades como principales productoras de revistas científicas. Si bien es muy difícil comparar los resultados para el resto de tipos de entidades propietarias, debido a la variedad de categorizaciones utilizadas para dicha variable, cabe mencionar el estudio de Rodrigues y Abadal (2014) en el que se identifica también a las asociaciones profesionales, junto a las universidades, como los principales productores de revistas científicas españolas.

Atendiendo a la rama de conocimiento, la producción de revistas científicas es mayor en el ámbito de las Artes y Humanidades y las Ciencias Sociales, que conjuntamente aglutinan el 68\% del total. A pesar de las diferencias metodológicas de las investigaciones de referencia, la mayoría coincide en que las revistas en dichas áreas ocupan los primeros puestos del total de la producción de revistas españolas. No así en los resultados de Rodrigues y Abadal (2014), donde el total de revistas de Medicina supera al de Ciencias Sociales y Humanidades. No obstante, debe tenerse en cuenta que dicho estudio no observa el conjunto de revistas científicas españolas, sino únicamente las indexadas en los informes de 2011 de Web of Science y Scopus.

El estudio también ha puesto de relieve que la mayoría de las revistas se publican en ambos formatos (50\%), cerca de un $30 \%$ publica solo en formato digital y un $20 \%$ de las revistas españolas se publican solo en formato impreso. Investigaciones previas auguraban cambios notables en el formato de publicación de las revistas científicas, apuntando principalmente a un incremento de las revistas digitales. Así, mientras Osca y otros (2008) informaron sobre un 40\% de revistas disponibles en formato digital, nuestros resultados revelaron que actualmente cerca del $80 \%$ de las revistas tiene versión electrónica. Asimismo, si se compara el resultado presentado en este estudio con el obtenido por Zamora y otros (2007), en el que el formato digital ascendía a un 9,3\% entre las revistas universitarias en el año 2005, se observa un claro aumento de este tipo de formato a lo largo de los últimos siete años.

Si bien no se recogieron datos que pudieran dar una respuesta concluyente sobre las razones que podrían estar frenando la migración definitiva al entorno digital, algunos editores apuntan al conflicto que supone para ellos renunciar a los acuerdos de intercambio o a los ingresos ligados al incentivo de ofrecer copias impresas de la revista (Claudio-González y Villarroya, 2015). De ahí la coexistencia de la versión papel y la digital, al permitirles también un abanico más amplio de fuentes complementarias de financiación (Melero y Abad-García, 2008). De hecho, la significación estadística obtenida al observar que el $69 \%$ de las revistas que percibieron ingresos por publicidad son 
las que publicaron tanto versiones impresas como digitales, apoya la hipótesis de que dichas revistas atraen más el interés de los anunciantes. En este sentido, los resultados de este estudio muestran que el perfil más frecuente de las revistas que recurren a los ingresos por publicidad corresponde a una revista del ámbito de las Ciencias de la Salud cuya entidad propietaria es una editorial comercial o una sociedad profesional y que se publica en ambos formatos.

La publicación de contenidos en inglés ha sido una estrategia muy debatida entre los editores nacionales. Desde quienes consideran el idioma como uno de los frenos a la internacionalización de las revistas científicas españolas (Buela Casal, 2001; Aréchaga, 2009; Fonseca-Mora y Aguaded, 2014) hasta quien hace ya quince años apuntaba directamente al problema lingüístico como responsable de que autores no anglosajones abandonaran las revistas de sus países de origen para publicar en revistas internacionales de mayor difusión en las bases de datos ISI Web of Knowledge (Urdín Caminos, 2001). Aunque en los resultados presentados apenas un 5\% de las revistas informó que publicaba solo en inglés, el total de las que informaron combinar el castellano con el inglés en proporciones variables ya supera el 40\%. Bordons y Gómez Caridad (1997) apuntaban a que, entre 1990 y 1993, cerca del 90\% de los artículos de autoría española incluidos en el Science Citation Index (SCI) y Social Sciences Citation Index (SSCI) estaban publicados en inglés, al tiempo que señalaban la escasez de revistas españolas en dichas bases de datos. Veinte años después, ya sea por reivindicación lingüística en favor de las lenguas nacionales, porque no es un asunto prioritario para los objetivos de determinadas revistas, o sencillamente por falta de recursos u otras barreras prácticas que dificultan la inclusión de contenidos en inglés, es evidente que la política lingüística mayoritaria entre las revistas españolas no se corresponde con la necesidad vigente de los autores de ampliar la visibilidad de sus artículos entre la comunidad científica internacional. En cualquier caso, la cuestión lingüística no es más que un aspecto entre muchos otros factores que influyen en la decisión de los autores españoles de publicar en inglés y/o en revistas extranjeras, que se escapan del alcance de esta investigación.

Según López-Torres Hidalgo (2015), el movimiento del acceso abierto emerge de la crisis en los costes de las publicaciones impresas, como reacción a la desproporcionada subida de precios de las revistas científicas en las pasadas décadas, y sobre todo de las oportunidades de acceso ofrecidas por internet. De ahí que la mayoría de las referencias a los aspectos económico-financieros de la edición de revistas científicas en la literatura del siglo XXI gire en torno al acceso abierto y los tipos de suscripción, más que al interés de diagnosticar, diseñar la estructura y planificar estrategias innovadoras para la dimensión económico-financiera de la actividad editorial desde una perspectiva más amplia e integradora. A modo de diagnóstico, los hallazgos presentados en relación a la financiación y la distribución de gastos y costes aportan un perfil general de la estructura económico-financiera de las revistas científicas en España y ayudan a identificar con mayores garantías de éxito factores clave que pueden afectar su sostenibilidad.

En un contexto de cambios en el sistema de comunicación científica, de recursos limitados y de experimentación con nuevos modelos en la estructura económico-financiera, se ha evidenciado que la estructura de ingresos de las revistas científicas en España se sostiene principalmente de las ayudas institucionales, ya sean subvenciones, ayudas de la entidad propietaria o ingresos en especie y, en menor medida, de patrocinios y donativos. Aun cuando el análisis ha puesto de relieve bastante heterogeneidad en la distribución de las distintas fuentes de ingresos, se observa una clara dependencia de las ayudas institucionales por más de la mitad de las revistas que las perciben. En el caso concreto de las revistas científicas universitarias, éstas se caracterizan por una marcada dependencia de las subvenciones de la Administración Pública y de las ayudas de la entidad propietaria para su subsistencia.

También se ha visto que cerca del $75 \%$ de las revistas que contaban con ingresos por suscripciones institucionales obtuvieron un resultado económico de equilibrio o beneficio. Habiéndose comprobado una relación estadísticamente significativa entre ambas variables, dicho resultado refuerza la idea de que los ingresos en concepto de suscripciones institucionales ofrecen mayores garantías de obtención de mejores resultados económicos.

Aunque podría registrarse un cambio significativo en el corto y medio plazo, el cobro de cargos por publicar es aún incipiente entre las revistas científicas españolas. Menos del 3\% de los que respondieron a la encuesta informó recibir ingresos por dicho concepto.

El análisis de los gastos ha puesto de relieve distribuciones más variadas entre las revistas, destacando el peso de los gastos en servicios externos, seguidos de los gastos de personal y de bienes corrientes. En cuanto a la distribución de los costes entre las distintas etapas del proceso de producción, más del 75\% de las revistas señala a los costes de producción como el principal coste de la revista.

En cuanto a la dimensión estratégica, los resultados muestran que el debate sobre las limitaciones y dificultades que afectan el desarrollo del acceso abierto sigue vigente, en la línea de lo comentado por otros autores en la literatura 
reciente (Alonso y Echevarría, 2014; García Arístegui y Rendueles, 2014). A pesar de que la experiencia de los editores con el acceso abierto es mayoritariamente positiva, el porcentaje de encuestados que aportaron una visión crítica y sincera sobre las limitaciones y dificultades a las que aún se enfrentan no ha sido marginal. Así, las áreas de mejora más señaladas por los encuestados fueron las limitaciones financieras y la débil estructura organizativa (Claudio-González y Villarroya, 2015).

Las limitaciones en la estructura organizativa de las revistas científicas españolas apuntan a que los editores se enfrentan al reto de lograr marcar una clara separación entre la dualidad de roles que asumen. Por un lado, su rol como editores les permite aportar su saber hacer gestionando la actividad científica. Pero, por otro lado, el rol administrativo/gerencial les requiere un esfuerzo adicional que compromete la eficiencia y consecución de la misión central del proyecto como medio de difusión de la producción científica. En definitiva, la gestión de una revista científica desde un modelo donde una misma persona ejerce ambos roles -editor científico y administrador- está alejado del llamado proceso de profesionalización de la revista. A ello se suma la fuerte dependencia que tienen las revistas científicas del trabajo voluntario para sostener su estructura organizativa (presente en un $70 \%$ de los encuestados), así como la exigua dedicación horaria del personal a cargo de las revistas (menos del 9\% del total de encuestados cuenta con personal propio a jornada completa). Dichas observaciones evidencian los pasos que quedan por recorrer hacia el camino de la profesionalización de la actividad editorial científica. Así, no es de extrañar que la ventaja competitiva que más editores valoraron como muy importante se relacione con las habilidades y competencias del equipo directivo.

El estudio aquí presentado deja abierta la puerta a futuras investigaciones. En este sentido, si bien el estudio incluyó una aproximación a la distribución porcentual de las principales categorías de ingresos, costes y gastos, un desglose más detallado -o bien cifras específicas de las categorías ya identificadas- aportarían información mucho más precisa de la dimensión económico-financiera. Una situación similar marca límites al conocimiento adquirido sobre la estructura organizativa de las revistas al no disponer de los datos exactos de la dedicación horaria de los recursos humanos con que cuentan las revistas, ahora recogida en intervalos horarios. Asimismo, requeriría especial atención ampliar las observaciones con variables que permitieran explorar otras áreas de gestión de las revistas. En particular, sobre las políticas editoriales y las acciones concretas que llevan a cabo los editores de cara a obtener buenos índices de calidad y un buen posicionamiento en los principales directorios y bases de datos de reconocimiento nacional e internacional.

\section{Agradecimientos}

Esta investigación se ha realizado en el marco del proyecto del Plan Nacional El acceso abierto (open access) a la ciencia en España: Análisis del grado de implantación y de la sostenibilidad de un nuevo modelo de comunicación cientifica, financiado por el Ministerio de Ciencia e Innovación (Referencia: CS02011-29503-C02-01).

\section{BIBLIOGRAFÍA}

ABAD-GARCÍA, F.; GONZÁLEZ-TERUEL, A.; ARGENTO, J. y RODRÍGUEZ-GAIRÍN, J.M. Características y visibilidad de las revistas españolas de ciencias de la salud en bases de datos. El Profesional de la Información, 2015, vol. 24, no 5, p. 537-550. http://doi.org/10.3145/epi.2015.sep.04.

ABADAL, E. y RIUS ALCARAZ, L. Revistas científicas de las universidades españolas: acciones básicas para aumentar su difusión e impacto. Revista Española de Documentación Científica, 2008, vol. 31, n ${ }^{\mathrm{o}}$ 2, p. $242-262$. http://doi.org/10.3989/redc.2008.v31.i2.427.

ABADAL, E.; OLLÉ CASTELLÀ, C.; ABAD-GARCÍA, F. y MELERO, R. Políticas de acceso abierto a la ciencia en las universidades españolas. Revista Española de Documentación Científica, 2013, vol. 36, n ${ }^{\circ}$ 2, p. 1-15. http://doi.org/10.3989/redc.2013.2.933.

ALONSO, A. y ECHEVARRÍA, J. Lucha de paradigmas: Leyes, ciencia y activismo en el mundo open. Argumentos de Razón Técnica, 2014, no 17, p. 21-38.

ARÉCHAGA, J. Internacionalización de las revistas científicas españolas: nuestra asignatura pendiente. Seminarios de la Fundación Española de Reumatología, 2009, vol. 10, n $^{\text {o }} \quad 4, \quad$ p. $109-111$. http://doi.org/10.1016/j.semreu.2009.09.001.

BJÖRK, B. y HEDLUND, T. Two scenarios for how scholarly publishers could change their business model to open access. Journal of Electronic Publishing, 2009, vol. 12, no 1, p. 1-10. http://doi.org/10.3998/3336451.0012.102.

BJÖRK, B.-C. y SOLOMON, D. Open access versus subscription journals: a comparison of scientific impact. $B M C$ Medicine, 2012, vol. 10, $\mathrm{n}^{\mathrm{o}} 1$, p. 73.

BORDONS, M. y GÓMEZ CARIDAD, I. La actividad científica española a través de indicadores bibliométricos en el período 1990-93. Revista General de Información y Documentación, 1997, vol. 7, nº 2, p. 69-86. 
BORDONS, M.; FELIPE, A. y GÓMEZ, I. Revistas científicas españolas con factor de impacto en el año 2000. Revista Española de Documentación Científica, 2002, vol. 25, nº 1, p. 49-73.

BUELA CASAL, G. La psicología española y su proyección internacional, el problema del criterio: internacional, calidad y castellano y/o inglés. Papeles del psicólogo, 2001, nº 79, p. 9.

CHI CHANG, C. Business models for open access journals publishing. Online Information Review, 2006, vol. 30, $\mathrm{n}^{\circ}$ 6, p. 699-713. http://doi.org/10.1108/14684520610716171.

CLAUDIO-GONZÁLEZ, M.G. y VILLARROYA, A. Desafíos de la edición de revistas científicas en acceso abierto. El Profesional de la Información, 2015, vol. 24, $\mathrm{n}^{\mathrm{o}} \quad$ 5, p. $517-525$. http://doi.org/http://dx.doi.org/10.3145/epi.2015.sep.02.

CROW, R. y GOLDSTEIN, H. Guide to Business Planning for Launching a New Open Access Journal. New York, USA: Open Society Institute, 2003.

CROW, R. Income Models for Open Access: An Overview of Current Practice. Washington, D.C.: Scholarly Publishing \& Academic Resources Coalition (SPARC), 2009.

DAI, N. et al. Publishing in open access era: focus on respiratory journals. Journal of Thoracic Disease, 2014, vol. 6, no. 5, p. 564-567. Disponible en: <https://www.ncbi.nlm.nih.gov/pmc/articles/PMC4014999/>. [Consulta: 7 de diciembre de 2016].

DAS, A.-K. Open Access: History and Developments. En: UNESCO (ed.), Introduction to Open Access [Open Access for Library Schools, 1]. Paris: UNESCO, 2015, p. 17-30.

DILLMAN, D.A. et al. Response rate and measurement differences in mixed-mode surveys using mail, telephone, interactive voice response (IVR) and the Internet. 2009. Social Science Research, 2009, vol. 38, p. 1-18.

FONSECA-MORA, M. y AGUADED, I. Las revistas científicas como plataformas para publicar la investigación de excelencia en educación: estrategias para atracción de investigadores. RELIEVE - Revista Electrónica de Investigación y Evaluación Educativa, 2014, vol. 20, nº 2, p. 1-11. http://doi.org/10.7203/relieve.20.2.4274.

FRIEND, F. Open access business models for research funders and universities. Copenhagen: Knowledge Exchange \& SPARC, 2011.

GARCÍA, A.M. ¿Qué aporta el acceso libre? Poca cosa, los principales problemas siguen y aparecen algunos más. Gaceta sanitaria, 2015, vol. 29, n 2, p. 142-144. http://doi.org/10.1016/j.gaceta.2014.11.008.

GARCÍA ARÍSTEGUI, D. y RENDUELES, C. Abierto, libre y público: Los desafíos políticos de la ciencia abierta. Argumentos de razón técnica: Revista Española de Ciencia, Tecnología y Sociedad, y Filosofía de la Tecnología, $2014, \mathrm{n}^{\mathrm{o}} 17$, p. $45-64$.

HOUGHTON, J.W. et al. Economic implications of alternative scholarly publishing models: Exploring the costs and benefits. Victoria \& Loughborough: JISC, 2009.

LAMBERTI, A.M. y SOLARO, G.N. Open access: un nuevo camino para recorrer en la sociedad del conocimiento. Disponible en: <http://eprints.rclis.org/17265/>. [Consulta: 21 de mayo de 2015].

LÓPEZ-TORRES HIDALGO, J. "Pagar por publicar" en revistas científicas. Revista Clínica de Medicina de Familia, 2015, vol. 8, no 3, p. 179-181. http://doi.org/10.4321/S1699-695X2015000300001.

MELERO, R. y ABAD-GARCÍA, F. Revistas open access: características, modelos económicos y tendencias. Textos Universitarios de Biblioteconomia i Documentació, 2008, $\mathrm{n}^{\circ} 20$.

MELERO, R. et al. Dulcinea: Iniciativa para el análisis de los derechos de copyright y autoarchivo de revistas científicas españolas. En: XI Jornadas Españolas de Documentación. Zaragoza: Fesabid, 2009.

MORRISON, H. Economics of scholarly communication in transition. First Monday, 2013, vol. $18, \mathrm{n}^{\mathrm{o}} 6$. http://doi.org/10.5210\%2Ffm.v18i6.4370.

OSCA-LLUCH, J. et al. Difusión y factor de impacto nacional e internacional de las revistas científicas españolas. Anales de Documentación, 2008, vol. 11, p. 145-164.

RIERA, M. y AIBAR, E. Does open access publishing increase the impact of scientific articles? An empirical study in the field of intensive care medicine. Medicina intensiva / Sociedad Española de Medicina Intensiva y Unidades Coronarias, 2013, vol. 37, nº 4, p. 232-240. http://doi.org/10.1016/j.medin.2012.04.002.

RODRIGUES, R.S. y ABADAL, E. Scientific journals in Brazil and Spain: Alternative publishing models. Journal of the Association for Information Science and Technology, 2014, vol. 65, $\mathrm{n}^{\mathrm{o}}$ 10, p. 2145-2151. http://doi.org/10.1002/asi.23115.

RODRÍGUEZ-YUNTA, L. y GIMÉNEZ-TOLEDO, E. Fusión, coedición o reestructuración de revistas científicas en humanidades y ciencias sociales. El Profesional de la Información, 2013, vol. 22, $\mathrm{n}^{\mathrm{o}}$ 1, p. 36-45. http://doi.org/10.3145/epi.2013.ene.05.

RODRÍGUEZ-YUNTA, L. Evaluación de las revistas españolas: pasos hacia un sistema que mida la apertura y la internacionalidad. Anuario ThinkEPI, 2015, vol. 9, p. 189-195. Disponible en: <http://eprints.rclis.org/28456/>. [Consulta: 7 de diciembre de 2016].

SCHIMMER, R.; GESCHUHN, K.K. y VOGLER, A. Disrupting the subscription journals' business model for the necessary large-scale transformation to open access. Max Planck Digital Library. Disponible en: http://hdl.handle.net/11858/00-001M-0000-0026-C274-7 doi:10.17617/1.3 [Consulta: 11 de abril de 2016]. 
URDÍN CAMINOS, C. La edición de revistas científicas en España. En: Román-Román, A. (coord.) La edición de revistas científicas: guía de buenos usos. Centro de Información y Documentación Científica (Cindoc - CSIC). Madrid, 2001, p. 11-16.

VILlARROYA, A.; CLAUDiO-GONZÁlEZ, M.G.; ABADAL, E. y MELERO, R. Modelos de negocio de las editoriales de revistas científicas: implicaciones para el acceso abierto. El Profesional de la Información, 2012, vol. 21, $\mathrm{n}^{\mathrm{o}}$ 2, p. 129-135. http://doi.org/http://dx.doi.org/10.3145/epi.2012.mar.02.

ZAMORA, H.; AGUILlO, I.; ORTEGA, J.L. y GRANADINO, B. Calidad formal, impacto y visibilidad de las revistas electrónicas universitarias españolas. El Profesional de la Información, 2007, vol. 16, nº 1, p. 13-23. 


\section{Apéndice A}

Variables observadas en cada una de las dimensiones del modelo de negocio

\begin{tabular}{|c|c|c|c|}
\hline \\
\hline & Parámetros & Categorías generales & Variables específicas \\
\hline \multirow{7}{*}{$\begin{array}{l}\text { D } \\
\text { i } \\
\text { m }\end{array}$} & \multirow{17}{*}{ 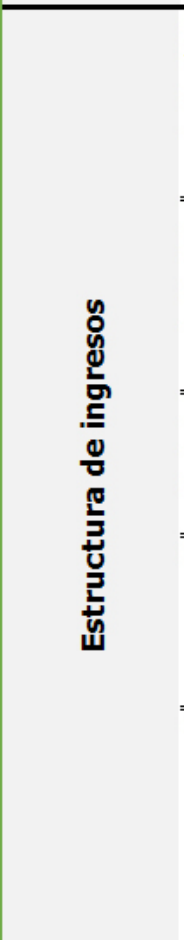 } & \multirow{4}{*}{$\begin{array}{l}\text { Ingresos monetarios generados de } \\
\text { transacciones directas con los } \\
\text { clientes o usuarios, ya sean lectores, } \\
\text { clientes institucionales o autores }\end{array}$} & Suscripciones individuales \\
\hline & & & Suscripciones institucionales \\
\hline & & & Pagos por uso \\
\hline & & & Cuotas por publicar \\
\hline & & \multirow{4}{*}{$\begin{array}{l}\text { Ingresos monetarios procedentes de } \\
\text { la administración pública u otras } \\
\text { entidades que apoyan la producción } \\
\text { científica }\end{array}$} & Ayudas de la entidad propietaria \\
\hline & & & Subvenciones de la administración pública \\
\hline & & & Patrocinios privados \\
\hline e & & & Donativos \\
\hline \multirow{4}{*}{$\begin{array}{l}\text { s } \\
\text { i } \\
6 \\
\text { n }\end{array}$} & & \multirow{3}{*}{$\begin{array}{l}\text { Ingresos monetarios procedentes de } \\
\text { otras transacciones comerciales } \\
\text { secundarias a la actividad principal }\end{array}$} & Publicidad \\
\hline & & & Co-organización de conferencias o congresos \\
\hline & & & Consultorías y servicios a terceros \\
\hline & & \multirow[t]{2}{*}{ Ingresos en especie } & $\begin{array}{l}\text { Apoyo administrativo (secretariado, servicios contables } \\
\text { y/o legales, etc.) } \\
\text { Equipos informáticos, material fungible, suministros, ...) }\end{array}$ \\
\hline $\mathbf{e}$ & & & Espacios (despachos, mobiliario, ...) \\
\hline $\mathbf{c}$ & & \multirow[t]{4}{*}{ Otros ingresos } & Cuotas de asociados \\
\hline $\mathbf{0}$ & & & Co-ediciones u otros intercambios \\
\hline $\mathbf{n}$ & & & Ventas directas \\
\hline ́́ & & & $\begin{array}{l}\text { Apoyo privado puntual (por ejemplo, publicidad } \\
\text { esporádica en función de un artículo concreto) }\end{array}$ \\
\hline i & \multirow{4}{*}{ 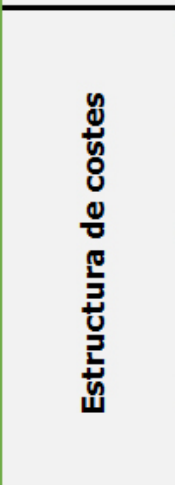 } & Creación de contenidos & $\begin{array}{l}\text { \% aproximado de todos los costes asociados a la } \\
\text { preparación del contenido editorial de la publicación }\end{array}$ \\
\hline - & & Producción & $\begin{array}{l}\text { \% aproximado de todos los costes asociados a la } \\
\text { maquetación (digital o papel), impresión, } \\
\text { encuadernación, etc. }\end{array}$ \\
\hline $\begin{array}{l}\mathbf{f} \\
\mathbf{i} \\
\mathbf{n}\end{array}$ & & "Gestión editorial & $\begin{array}{l}\% \text { aproximado de todos los costes de comercialización, } \\
\text { ventas de publicidad, finanzas y administración, incluidos } \\
\text { los gastos de gestión y de las oficinas que llevan a cabo } \\
\text { dichas actividades }\end{array}$ \\
\hline $\begin{array}{l}\mathbf{a} \\
\mathbf{n} \\
\mathbf{c}\end{array}$ & & Distribución & $\begin{array}{l}\text { \% aproximado de los costes de distribución, incluyendo } \\
\text { todos los costes de mantenimiento de archivos de } \\
\text { abonados y el servicio al cliente }\end{array}$ \\
\hline \multirow{5}{*}{$\begin{array}{l}\mathbf{e} \\
\mathbf{r} \\
\mathbf{a}\end{array}$} & \multirow{4}{*}{ 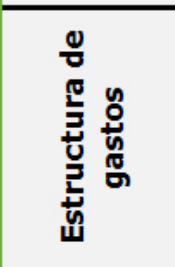 } & Personal & $\%$ aproximado de los gastos de personal \\
\hline & & Revisores externos & \% aproximado de pagos a revisores externos \\
\hline & & Bienes corrientes & $\begin{array}{l}\text { \% aproximado de los gastos en materias primas y otros } \\
\text { aprovisionamientos y suministros }\end{array}$ \\
\hline & & Bienes de capital fijo & $\begin{array}{l}\text { \% aproximado de los gastos de reposición de } \\
\text { equipamientos, infraestructuras, etc. }\end{array}$ \\
\hline & 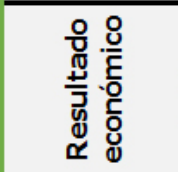 & $\begin{array}{l}\text { Resultado económico obtenido: } \\
\text { Beneficio; Pérdidas, Equilibrio }\end{array}$ & Identificación del balance de tres años consecutivos \\
\hline
\end{tabular}


Apéndice A (continuación).

Variables observadas en cada una de las dimensiones del modelo de negocio

\begin{tabular}{|c|c|c|c|}
\hline & & Dimensión operativ & \\
\hline & Parámetros & Categorías generales & Variables específicas \\
\hline & " & Personal propio (empleados remunerados) & Total de personas por cada tipo de dedicación horaria \\
\hline & ह్ & Personal voluntario (no remunerado) & _Dedicación parcial mínima \\
\hline & 点 & $\begin{array}{l}\text { Personal externo contratado puntualmente según las } \\
\text { necesidades }\end{array}$ & $\begin{array}{l}\text { (menos de } 10 \text { horas semanales) } \\
\text { Dedicación media }\end{array}$ \\
\hline $\mathbf{p}$ & ö & Personal becario & (entre 10 y 25 horas semanales) \\
\hline $\mathrm{m}$ e & $\overline{\bar{z}}$ & & $\begin{array}{l}\text {-Dedicacion completa } \\
\text { (más de } 25 \text { horas semanales) }\end{array}$ \\
\hline $\mathbf{r}$ & & & \\
\hline $\begin{array}{ll}\mathrm{a} \\
\mathrm{s} & \mathrm{t}\end{array}$ & 胥 & $\begin{array}{l}\text { Gestión editorial: dirección y administración editorial de } \\
\text { la revista }\end{array}$ & Total de personas por cada actividad editorial \\
\hline ó $\mathbf{v}$ & 宽 & $\begin{array}{l}\text { Creación de contenidos: preparación del contenido } \\
\text { editorial de la publicación }\end{array}$ & \\
\hline a & 흠 & $\begin{array}{l}\text { Producción: maquetación digital / papel, impresión, } \\
\text { encuadernación, técnicos de diseño, informáticos, } \\
\text { correctores, etc. }\end{array}$ & \\
\hline & $\frac{n}{d} \bar{a}$ & $\begin{array}{l}\text { Distribución: incluye todas las actividades de distribución } \\
\text { y comercialización de la revista }\end{array}$ & \\
\hline & & Dimensión estratégi & \\
\hline & Parámetros & Categorías generales & Variables específicas \\
\hline & g & $\begin{array}{l}\text { a. La modificación en los procesos de acreditación y } \\
\text { promoción en las instituciones de educación superior }\end{array}$ & Frecuencia de las valoraciones \\
\hline & N & b. La expansión de los mercados emergentes & Nivel de importancia para cada una (escala de Likert): \\
\hline D & $\sum_{\overparen{\pi}}^{0}$ & $\begin{array}{l}\text { c. El desarrollo de las tecnologías de la información y la } \\
\text { comunicación }\end{array}$ & _Oportunidad menor \\
\hline $\mathbf{m}$ & $\frac{8}{y}$ & $\begin{array}{l}\text { d. Cambios en las necesidades o en los hábitos de los } \\
\text { usuarios de la revista }\end{array}$ & $\begin{array}{l}\text {-Amenaza menor } \\
\text { Gran amenaza }\end{array}$ \\
\hline $\begin{array}{l}\mathbf{e} \\
\mathbf{n}\end{array}$ & $\stackrel{\frac{\pi}{2}}{5}$ & $\begin{array}{l}\text { e. Las modificaciones en las normativas en relación al } \\
\text { acceso abierto a los datos y a las publicaciones científicas }\end{array}$ & \\
\hline s & t! & $\begin{array}{l}\text { f. El aumento en la competencia de los actores estables } \\
\text { g. La liberalización del comercio } \\
\text { h. La presencia de grandes multinacionales en el sector }\end{array}$ & \\
\hline $\mathbf{n}$ & & a. Calidad el servicio al cliente & Frecuencia de las valoraciones \\
\hline & & b. Habilidades y competencias del equipo directivo & Nivel de importancia para cada una (escala de Likert): \\
\hline e & & c. Prestigio comité editorial & _Muy importante \\
\hline 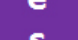 & $\stackrel{0}{ \pm}$ & d. Habilidades y competencia de los empleados & _Bastante importante \\
\hline $\mathbf{s}$ & \pm & e. Valor de la propiedad intelectual & $\begin{array}{l}\text { _Importancia moderada } \\
\text { Poco importante }\end{array}$ \\
\hline $\mathbf{t}$ & $\cong$ & f. Factor de impacto & _Nada importante \\
\hline $\mathbf{r}$ & है & g. Periodicidad & \\
\hline $\mathbf{a}$ & $\tilde{u}_{n}$ & h. Bajos costes de producción & \\
\hline $\mathbf{t}$ & 党 & i. Precios competitivos & \\
\hline é & $\stackrel{\Xi}{E}$ & j. $\quad$ Prestigio de la editorial & \\
\hline & $\stackrel{\bar{D}}{>}$ & k. Relación con los proveedores & \\
\hline g & & I. Número citas & \\
\hline I & & m. Celeridad revisión & \\
\hline c & & n. Rapidez publicación & \\
\hline a & $\overline{0}$ & Experiencia con la publicación en Acceso Abierto & Frecuencias (sí / no) \\
\hline & 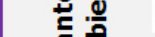 & Valoración general de la experiencia & Respuestas abiertas \\
\hline & 象定 & $\begin{array}{l}\text { Dificultades para adoptar la filosofía de Acceso Abierto: } \\
\text { limitaciones financieras, limitaciones en la estructura } \\
\text { organizativas o limitaciones tecnológicas }\end{array}$ & Frecuencias \\
\hline & & Otras dificultades no especificadas & Respuestas abiertas \\
\hline
\end{tabular}

